\title{
Un Nuevo Anovulador Antiandrogénico
}

Dres. A. Millet-Part, A. Blanes-Espi, J. Ferrer-Barriendos, E. Acosta-Cajiao* y L. Siqueira**

Facultad de Medicina de Valencia (España)

Cátedra de Obstetricia y Ginecología Profesor F. Bonilla-Musoles.

\section{INTRODUCCION}

El hirsutismo, la seborrea, el acné y la alopecia son síntomas bien de una sobreproducción de andrógenos de origen suprarrenal u ovárico, bien de una hipersensibilidad del folículo piloso $(22,25$, 26).

Una acción antiandrógena intensa se logra con el acetato de ciproterona (Androcur), que es también un potente progestágeno y antigonadotropo $(5,8$, $16,28)$. Tal preparado ha sido muy usado en el tratamiento de estos síntomas sobre todo en países de habla alemana y española, habiendo sido utilizado apenas en los de habla inglesa; en los Estados Unidos no ha sido autorizado su empleo por la F.D.A. A este preparado se le añade hoy un estrógeno el etinil-

* Facultad de Medicina Universidad del Rosario. Bogotá (Colombia)

Hospital e Maternidade de Nossa Senhora Das Gracas. Pres. Prudente (Brasil) estradiol, para neutralizar la acción progesterónica y en consecuencia amenorréica del Androcur. Es comercializado con el nombre de Diane.

Es muy frecuente que en nuestra consulta ginecológica nos enfrentemos con el hirsutismo, seborrea, acné y alopecia mencionados, pues las pacientes que presentan esta sintomatología androgénica aquejan ordinariamente trastornos menstruales; también acuden remitidas por el departamento de Dermatología tras el fracaso de las terapeúticas a que fueron sometidas (corticoides, antibióticos, queratolíticos, etc), permitiendo recuperar una amplia casuística, que compararemos a continuación.

Diversos esquemas terapeúticos hemos empleado en estas pacientes.

1. Método continuo (29) (Androcur). Administración continua de acetato de Ciproterona (A.C) a dosis variables de 25 a 150 mgrs con descansos cada 3 meses. 
2. Combinado con altas dosis (Androcur $50 \mathrm{mg} \mathrm{0,05} \mathrm{mg}$ de Etinil estradiol), variables, en ciclos de 21 días.

3. Combinado con bajas dosis (Diane: $2 \mathrm{mgr}$ de A.C. y $0.05 \mathrm{mgr}$ de E.E.I, en ciclos de 21 días.

Queremos hacer aquí un estudio comparativo de estas metódicas, recalcando en sus resultados clínicos, así como destacar la acción anticonceptiva del método combinado de bajas dosis (Diane), que lógicamente divulgará mucho su empleo.

\section{Material y Métodos}

Está constituido por 54 pacientes tratadas con el método combinado de dosis bajas (Diane); la mayoría habían sido previamente tratadas con método continuo a dosis variables de Acetato de Ciproterona, y/o método combinado de altas dosis (Tabla No. 1).

\section{Tabla No. 1}

\section{DISTRIBUCION DE LAS PACIENTES Y NUMERO DE CICLOS SEGUN EL TIPO DE TRATAMIENTO}

\begin{tabular}{|c|c|c|c|c|c|c|}
\hline \multirow{2}{*}{$\begin{array}{c}\text { Tipos de } \\
\text { Medicación }\end{array}$} & \multicolumn{4}{|c|}{ Acetato de Ciproterone } & \multicolumn{2}{|c|}{ Combinado } \\
\hline & $25 \mathrm{mg}$. & $50 \mathrm{mg}$. & $100 \mathrm{mg}$. & $150 \mathrm{mg}$. & C/E. & Diane \\
\hline $\begin{array}{l}\text { No. Total de } \\
\text { Ciclos } \\
\text { No. Total de } \\
\text { pacientes }\end{array}$ & $\begin{array}{l}6 . \\
2 .\end{array}$ & $\begin{array}{r}27 . \\
7 .\end{array}$ & $\begin{array}{r}100 . \\
16 .\end{array}$ & $\begin{array}{l}47 . \\
10 .\end{array}$ & $\begin{array}{c}205 . \\
31 .\end{array}$ & $\begin{array}{r}250 . \\
54 .\end{array}$ \\
\hline
\end{tabular}

* Acetato de Ciproterona más etinil es tradiol (combinado de altas dosis)

Siete pacientes habian tomado previamente $A C$ en dosis variables de 25,50 , 100 y $150 \mathrm{mg}$ diarios durante períodos de tiempo que oscilaban entre 3 y $10 \mathrm{ci}$ clos (considerando como ciclos enteros períodos de un mes de toma), y Diane de 2 a 6 ciclos (Tabla No. 2).

Tabla No. 2

\section{CICLOS DE PACIENTES TRATADAS CON ACETATO DE CIPROTERONA Y DIANE}

\begin{tabular}{cccccc}
\hline Casos & $25 \mathrm{mg}$. & $50 \mathrm{mg}$. & $100 \mathrm{mg}$. & $150 \mathrm{mg}$. & Diane \\
\hline 1. & 3. & - & - & - & 5 \\
2. & 3. & 6. & 10 & - & 6 \\
3. & - & - & 3 & - & 4 \\
4. & - & - & - & 4 & 3 \\
5. & - & - & 6. & 3 & 2 \\
6. & - & 3. & - & - & 6 \\
7. & - & - & 7 & - & 2 \\
\hline No. Total & 6. & 9. & 26 & 7 & 28 \\
\hline
\end{tabular}

Dieciseis pacientes habían tomado previamente $A C$ en dosis variables de 50,100 y $150 \mathrm{mg}$ diarios durante períodos de tiempo que oscilaban entre $3 y$ 14 ciclos, seguido de método combinado de altas dosis en períodos de tiempo que oscilan entre 3 y 12 ciclos, y Diane de 1 a 7 ciclos (Tabla No. 3).

16 pacientes habían tomado previamente método combinado de altas dosis en períodos de tiempo que oscilaron entre 2 y 22 ciclos, y Diane de 3 a 7 ciclos, (Tabla No. 4).

Con nuestra metódica exploratoria (27) hemos clasificado a las pacientes en:

- Hirsutismo idiopático

10 casos.

- Hirsutismo suprarrenal 11 casos.

- Hirsutismo ovárico

25 casos.

- Hirsutismo Yatrogénico 2 casos.

- Acné

24 casos.

- Seborrea

37 casos.

- Alopecia 
Tabla No. 3

\section{PACIENTES TRATADAS CON A.C., DESPUES CON C + E Y POR ULTIMO CON DIANE}

\begin{tabular}{|cccccc|}
\hline Casos & $50 \mathrm{mg}$. & $100 \mathrm{mg}$. & $150 \mathrm{mg}$ & $\mathrm{C}+\mathrm{E}$ & Diane \\
\hline 1 & - & 3 & - & 3 & 3 \\
2 & 3 & 9 & 3 & 8 & 6 \\
3 & - & - & 6 & 12 & 6 \\
4 & - & 6 & 6 & 9 & 3 \\
5 & - & 6 & - & 3 & 3 \\
6 & - & 13 & - & 3 & 4 \\
7 & 3 & 14 & - & 12 & 3 \\
8 & - & - & 5 & 6 & 6 \\
9 & 6 & - & - & 5 & 6 \\
10 & 3 & 5 & - & 6 & 6 \\
11 & 3 & 3 & 6 & 3 & 6 \\
12 & - & - & 3 & 5 & 7 \\
13 & - & 3 & - & 1 & 1 \\
14 & - & 6 & - & 3 & 6 \\
15 & - & 3 & 8 & 6 & 6 \\
16 & - & 3 & 3 & 11 & 5 \\
\hline No. Total & & & & & \\
de ciclos & 18 & 74 & 40 & 96 & 78 \\
\hline
\end{tabular}

Tabla No. 4

\begin{tabular}{rccrc}
\hline Casos & Diane & $\begin{array}{c}\text { No. de } \\
\text { Casos }\end{array}$ & C + E Diane \\
\hline 1 & 5 & 1 & 5 & 3 \\
2 & 7 & 2 & 8 & 7 \\
3 & 7 & 3 & 6 & 5 \\
4 & 9 & 4 & 22 & 6 \\
5 & 4 & 5 & 4 & 6 \\
6 & 5 & 6 & 3 & 5 \\
7 & 4 & 7 & 2 & 3 \\
8 & 3 & 8 & 8 & 4 \\
9 & 2 & 9 & 2 & 5 \\
10 & 2 & 10 & 16 & 7 \\
11 & 3 & 11 & 6 & 6 \\
12 & 3 & 12 & 10 & 6 \\
13 & 2 & 13 & 6 & 6 \\
14 & 4 & 14 & 9 & 5 \\
15 & 3 & 15 & 2 & 5 \\
16 & 2 & & & \\
\hline
\end{tabular}

No. Total

No. Total

de Ciclos

65

$79 \quad 109$

Los grados del hirsutismo fueron clasificados, según el método de Lorenzo y Ferriman, en Grado I: leve, Grado 11: moderado, y Grado III: severo, $(12,23)$.

En cuanto al acné nuestro criterio para clasificarlo ha sido:

- Leve: acné papuloso y acné comedoniano.

-- Moderado: acné papulopustuloso, quístico y polimorfo.

- Grave: acné conglobata (cara, espalda, hombros, ...)

\section{RESULTADOS}

\section{Acción anticonceptiva}

En aquellas pacientes que nos referían relaciones sexuales normales y tomaban el Diane con finalidad terapéutica para sus síntomas secundarios androgénicos, nos encontramos con 19 pacientes que habían tomado 96 ciclos de Diane sin presentar embarazo, hecho que está de acuerdo con lo reportado en la mayoría de la literatura $(7,17,19)$.

\section{Control del ciclo}

1. Con Ciproterona: en 23 mujeres con dosis continuas lo evidente fue una amenorrea en el $69.5 \%$ y algún manchado esporádico en el $30.5 \%$.

2. Con C. + E.: en 10 mujeres eumenorréicas, con un total de 70 ciclos, obtuvimos un $51.4 \%$ de eumenorreas, $20 \%$ de amenorreas, $11.4 \%$ de eumenorreas alternando con amenorreas, y $17.1 \%$ de hipomenorreas.

En 6 mujeres con opsomenorreas, en 28 ciclos, en $50 \%$ se obtuvo eumenorrea y en el otro $50 \%$ eumenorreas alternando con amenorreas. 
Tabla No. 5

\section{DIAGNOSTICOS}

\begin{tabular}{|l|c|c|c|c|r|r|r|}
\hline \multirow{2}{*}{ Intensidad } & \multicolumn{3}{|c|}{ Hirsutismo } & \multirow{2}{*}{ ACNE } & Seborrea & Alopecia \\
\cline { 2 - 5 } & Idiopático & Suprarrenal & Ovárico & Yatrogénico & & & \\
\hline Leve & 6 & 3 & 11 & 2 & 9 & 22 & 7 \\
Moderado & 4 & 6 & 12 & - & 11 & 11 & 2 \\
Severo & 0 & 2 & 2 & - & 4 & 4 & 1 \\
\hline No. Total & 10 & 11 & 25 & 2 & 24 & 37 & 10 \\
de Casos & 10 & & & & & \\
\hline
\end{tabular}

En 11 mujeres con oligomenorrea en 88 ciclos, $23.2 \%$ presentaron eumenorrea, $16.3 \%$ amenorrea, $46.4 \%$ eumenorrea alternando con amenorrea, y $14 \%$ hipomenorrea.

En 4 mujeres con amenorrea secundaria con un total de 19 ciclos, $71 \%$ presentaron eumenorrea, $10.5 \%$ amenorrea, y $15.7 \%$ una hipomenorrea (ver Tabla No. 6).

3. Con Diane: 13 mujeres eumenorréicas, con un total de 65 ciclos, mos- traron eumenorrea en el $78.4 \%$ y en el $21.5 \%$ hubo una discreta hipomenorrea.

En una paciente con amenorrea primaria hipogonadotropa, en 3 ciclos se produjo eumenorrea en los tres.

En 15 mujeres con opsomenorreas en 72 ciclos, las reglas fueron normales en el $84.5 \%$ y en un $15.4 \%$ hubo una discreta hipomenorrea.

En 14 mujeres con oligomenorreas con un total de 70 ciclos, en el $52.6 \%$

Tabla No. 6

CONTROL DEL CICLO CON C + E

\begin{tabular}{|l|c|c|c|c|}
\hline Diagnóstico & \multirow{2}{*}{ Eumenorrea } & Opsomenorrea & Oligomenorrea & $\begin{array}{l}\text { Amenorrea } \\
\text { Secundaria }\end{array}$ \\
\cline { 1 - 1 } Resultados & & $14(50 \%)$ & $20(23.2 \%)$ & $14(71 \%)$ \\
\cline { 1 - 2 } Eumenorrea & $36(51.4 \%)$ & - & $14(16.3 \%)$ & $3(10.5 \%)$ \\
Amenorrea & $14(20 \%)$ & - & $40(46.4 \%)$ & $2(15.7 \%)$ \\
EU-Amenorrea & $8(11.4 \%)$ & $14(50 \%)$ & $12(14 \%)$ & $2 \%$ \\
Hipomenorrea & $12(17.1 \%)$ & - & 88 & 19 \\
\hline $\begin{array}{l}\text { No. Total } \\
\text { de Ciclos }\end{array}$ & 70 & & & \\
\hline
\end{tabular}


Tabla No. 7

CONTROL DEL CICLO CON DIANE

\begin{tabular}{|l|c|c|c|c|c|}
\hline $\begin{array}{l}\text { Diagnóstico } \\
\text { Resultados }\end{array}$ & Eumenorrea & Opsomenorrea & Oligomenorrea & $\begin{array}{c}\text { Amenorrea } \\
\text { Secundaria }\end{array}$ & $\begin{array}{c}\text { Amenorrea } \\
\text { Primaria }\end{array}$ \\
\hline $\begin{array}{l}\text { Eumenorrea } \\
\text { Hipomeno- } \\
\text { rrea } \\
\begin{array}{l}\text { EU. Ameno- } \\
\text { rrea }\end{array}\end{array}$ & $\begin{array}{c}51(78.4 \%) \\
14(21.5 \%)\end{array}$ & $11(15.4 \%)$ & $30(42.8 \%)$ & $3(7.5 \%)$ & - \\
$\begin{array}{l}\text { No. Total } \\
\text { de Ciclos }\end{array}$ & 65 & - & $3(4.2 \%)$ & $3(7.5 \%)$ & - \\
\hline
\end{tabular}

obtuvimos eumenorreas, $42.8 \%$ hipomenorreas y un $4.2 \%$ eumenorreas alternando con amenorreas.

\section{Control del hirsutismo}

1. Con acetato de Ciproterona han sido tratadas 34 pacientes, de las cuales 7 han recibido dosis de $50 \mathrm{mg} / \mathrm{d}$ ía, obteniendo en $42.8 \%$ mejoría leve, en $28.5 \%$ mejoría buena y en $28.5 \%$ mejoría marcada.

Con $100 \mathrm{mg} / \mathrm{d}$ ía se han estudiado 16 pacientes obteniendo un $18.7 \%$ de fracasos, $25 \%$ de buena mejoría y $56.2 \%$ de mejoría marcada.

Con $150 \mathrm{mg} / \mathrm{d}$ ía se han estudiado 11 pacientes obteniendo $18.1 \%$ de buena mejoría y $63.6 \%$ de mejoría marcada.

En conjunto se han obtenido $17.7 \%$ de fracasos, $8.8 \%$ de mejoría leve, $23.5 \%$ de buena mejoría y $52.6 \%$ de mejoría marcada. En total, mejoría de algún tipo en $85.2 \%$ de los casos.

2. Con $C$. + E. han sido tratadas 32 pacientes obteniéndose un $18.7 \%$ de fracasos, $6.2 \%$ de mejoría leve, $18.7 \%$ de buena mejoría, y $56.2 \%$ de mejoría marcada. Mejoría de algún tipo en el $81.2 \%$.

3. Con Diane han sido tratadas 50 pacientes, con $26 \%$ de fracasos, $18 \%$ de buena mejoría y $38 \%$ de mejoría marcada. Mejoría de algún tipo en el $74 \%$ (Tabla No. 8 ).

\section{Control del acné}

1. Con acetato de Ciproterona han sido tratadas 22 pacientes, de las cuales 4 con dosis de $50 \mathrm{mg}$, obteniendo $25 \%$ mejoría leve, $25 \%$ buena mejoría y $50 \%$ mejoría marcada.

Con dosis de $100 \mathrm{mg}$ han sido tratadas 11 pacientes, obteniendo $18.1 \%$ de buena mejoría y $81.8 \%$ de mejoría marcada.

Con dosis de $150 \mathrm{mg}$ han sido tratadas 7 pacientes, obteniendo el $100 \%$ de mejoría marcada.

En conjunto se aprecia un $4.5 \%$ de mejoría leve, $13.5 \%$ de buena mejoría y 81.9 de mejoría marcada; mejoría de algún tipo en el $100 \%$. 
Tabla No. 8

CONTROL DEL HIRSUTISMO CON ACETATO

DE CIPROTERONA C +E. Y DIANE

\begin{tabular}{|c|c|c|c|c|c|}
\hline Resultados & $(-)$ & + & ++ & +++ & $\begin{array}{c}\text { No. Total de } \\
\text { Pacientes }\end{array}$ \\
\hline $50 \mathrm{mg}$. & 0 & $3(42.8 \% / \mathrm{o})$ & $2(28.5 \%)$ & $2(28.5 \%)$ & 7 \\
$100 \mathrm{mg}$. & $3(18.7 \% \mathrm{o})$ & 0 & $4(25 \%)$ & $9(56.2 \%)$ & 16 \\
$150 \mathrm{mg}$. & $2(18.1 \%)$ & 0 & $2(18.1 \% / 0)$ & $7(63.6 \%)$ & 11 \\
C.t E. & $6(18.7 \%)$ & $2(6.2 \%)$ & $6(18.7 \%)$ & $18(56.2 \%)$ & 32 \\
Diane & $13(26 \%)$ & $9(18 \%)$ & $9(18 \%)$ & $19(38 \%)$ & 50 \\
\hline
\end{tabular}

(-) Fracasos + Mejor f́a leve ++ Buena mejoría +++ Mejoría marcada

2. Con C. + E. han sido tratadas 9 pacientes, obteniendo un $11.1 \%$ de mejorías leves, $22 \%$ de buena mejoría y $66.6 \%$ de mejoría marcada; en conjunto se aprecia mejoría de algún tipo en el $100 \%$.
3. Con Diane han sido tratadas 24 pacientes, obteniendo un $12.5 \%$ de fracasos, $12.5 \%$ de buena mejoría y $75 \%$ de mejoría marcada; en conjunto $87.5 \%$ de mejoría de algún tipo (ver $\mathrm{Ta}$ bla No. 9).

Tabla No. 9

CONTROL DEL ACNE CON ACETATO DE CIPROTERONA, C + E Y DIANE

\begin{tabular}{|c|c|c|c|c|c|}
\hline Resultados & $(-)$ & + & ++ & +++ & $\begin{array}{c}\text { No. Total de } \\
\text { Pacientes }\end{array}$ \\
\hline $50 \mathrm{mg}$. & 0 & $1(25 \%)$ & $1(25 \%)$ & $2(50 \%)$ & 4 \\
$100 \mathrm{mg}$. & 0 & 0 & $2(18.1 \% / 0)$ & $9(81.8 \%)$ & 11 \\
$150 \mathrm{mg}$. & 0 & 0 & 0 & $7(100 \%)$ & 7 \\
C. $+\mathrm{E}$. & 0 & $1(11.1 \%)$ & $2(22.2 \%)$ & $6(66.6 \%)$ & 9 \\
Diane & $3(12.5 \%)$ & 0 & $3(12.5 \%)$ & $18(75 \%)$ & 24 \\
\hline
\end{tabular}




\section{Control de la seborrea}

1. Con acetato de Ciproterona se han estudiado 25 pacientes, 4 de las cuales con dosis de $50 \mathrm{mg}$, obteniendo mejoría leve en un $25 \%$ y $75 \%$ de mejoría marcada.

Con dosis de $100 \mathrm{mg}$ se estudiaron 12 pacientes, obteniendo $8.3 \%$ de mejoría leve, $16.6 \%$ de buena mejoría y $75 \%$ de mejoría marcada.

Con dosis de $150 \mathrm{mg}$ se estudian 9 pacientes, obteniendo $11.1 \%$ de mejoría leve y $88.8 \%$ de mejoría marcada.

En conjunto se obtiene una mejoría leve en $12 \%, 8 \%$ de buena mejoría $y$ $80 \%$ de mejoría marcada. Presentando algún tipo de mejoría el $100 \%$ de los casos.

2. Con C. + E. se estudian 20 pacientes, obteniendo $5 \%$ de fracasos, $10 \%$ de mejoría leve, $15 \%$ de buena mejoría y $70 \%$ de mejoría marcada, en conjunto se obtiene $95 \%$ de mejoría de alǵun tipo.

3. Con Diane se estudian 37 pacientes, obteniendo un $10.8 \%$ de fracasos, $2.7 \%$ de mejoría leve $13.5 \%$ de buena mejoría y $72.9 \%$ de mejoría marcada; en conjunto se obtiene mejoría de algún tipo en el $89.2 \%$ (ver Tabla No. 10).

\section{Control de la Alopecia}

1. Con acetato de Ciproterona se estudiaron 8 pacientes, de las cuales $33.3 \%$ presentó mejoría leve, $33.3 \%$ mejoría moderada, y $33.3 \%$ mejoría marcada, con dosis de $50 \mathrm{mg}$.

Con dosis de $100 \mathrm{mg}$ se obtiene un $100 \%$ de mejoría marcada.

Con dosis de $150 \mathrm{mg}$ se obtiene $33.3 \%$ de buena mejoría y $66.6 \%$ de mejoría marcada; en conjunto se obtiene mejoría leve $12.5 \%$ de los casos, mejoría buena en $25 \%$ y mejoría marcada en $62.5 \%$; presentando mejoría de algún tipo en $100 \%$.

2. Con C. + E. se estudian 5 pacientes, obteniendo un $40 \%$ de mejoría buena y $60 \%$ de mejoría marcada; en conjunto mejoría de algún tipo en el $100 \%$.

3. Con Diane se estudiaron 100 pacientes, obteniendo $10 \%$ de fracasos,

Tabla No. 10

CONTROL DE LA SEBORREA CON ACETATO DE CIPROTERONA, C + E Y DIANE

\begin{tabular}{|c|c|c|c|c|c|}
\hline Resultados & $(-)$ & + & ++ & +++ & $\begin{array}{c}\text { No. Total de } \\
\text { Paciences }\end{array}$ \\
\hline $50 \mathrm{mg}$. & 0 & $1(25 \%)$ & 0 & $3(75 \%)$ & 4 \\
$100 \mathrm{mg}$. & 0 & $1(8.3 \% / \mathrm{o})$ & $2(16.6 \%)$ & $9(75 \%)$ & 12 \\
$150 \mathrm{mg}$. & 0 & $1(11.1 \% / \mathrm{o})$ & 0 & $8(88.8 \%)$ & 9 \\
$\mathrm{C} .+\mathrm{E}$. & $1(5 \% / \mathrm{o})$ & $2(10 \%)$ & $3(15 \% / \mathrm{o})$ & $14(70 \% \mathrm{o})$ & 20 \\
Diane & $4(10.8 \%)$ & $1(2.7 \% / \mathrm{o})$ & $5(13.5 \%)$ & $27(72.9 \% / 0)$ & 37 \\
\hline
\end{tabular}


Tabla No. 11

\section{CONTROL DE ALOPECIA CON ACETATO DE CIPROTERONA, C + E Y DIANE}

\begin{tabular}{|c|c|c|c|c|c|}
\hline Resultados & $(-)$ & + & ++ & +++ & $\begin{array}{c}\text { No. Total de } \\
\text { Pacientes }\end{array}$ \\
\hline $50 \mathrm{mg}$. & 0 & $1(33.3 \%)$ & $1(33.3 \%)$ & $1(33.3 \%)$ & 3 \\
$100 \mathrm{mg}$. & 0 & 0 & 0 & $2(100 \%)$ & 2 \\
$150 \mathrm{mg}$. & 0 & 0 & $1(33.3 \%)$ & $2(66.6 \%)$ & 3 \\
$\mathrm{C} .+\mathrm{E}$. & 0 & 0 & $2(40 \%)$ & $3(60 \%)$ & 5 \\
Diane & $1(10 \%)$ & $1(10 \%)$ & $2(20 \%)$ & $6(60 \%)$ & 10 \\
\hline
\end{tabular}

$10 \%$ de mejoría leve, $20 \%$ de mejoría buena y $60 \%$ de mejoría marcada, presentando mejoría de algún tipo en el $90 \%$ (Tabla No. 11).

\section{Efectos Secundarios}

1. Con acetato de Ciproterona: en 23 pacientes se presentaron $8.6 \%$ de astenia, $4.3 \%$ de prurito nocturno y $4.3 \%$ de aumento de peso; en conjunto, en $30.1 \%$ se presentó algún tipo de efecto secundario, que solo en el $4.3 \%$ fue lo suficientemente molesto para justificar la suspensión de la medicación.

Tabla No. 12

\section{EFECTOS SECUNDARIOS}

\begin{tabular}{|c|c|c|c|}
\hline $\begin{array}{l}\text { Tipo de } \\
\text { Tratamiento }\end{array}$ & Alteraciones & $\begin{array}{l}\text { Número } \\
\text { de Casos }\end{array}$ & $\begin{array}{r}\text { Número de } \\
\text { Pacientes }\end{array}$ \\
\hline $\begin{array}{l}\text { Acetato de } \\
\text { Ciproterona }\end{array}$ & $\begin{array}{l}\text { Prurito nocturno } \\
\text { Depresión } \\
\text { Astenia } \\
\text { Intolerancia } \\
\text { Aumento de Peso }\end{array}$ & $\begin{array}{l}1(4.3 \%) \\
2(8.6 \%) \\
2(8.6 \%) \\
1(4.3 \%) \\
1(4.3 \%)\end{array}$ & 23 \\
\hline C. + E. + & $\begin{array}{l}\text { Molestias digestivas } \\
\text { Intolerancia total } \\
\text { Astenia } \\
\text { Frigidez }\end{array}$ & $\begin{array}{l}2(7.2 \%) \\
1(3.6 \%) \\
2(7.2 \%) \\
2(7.2 \%)\end{array}$ & 29 \\
\hline Diane & $\begin{array}{l}\text { Terisión mamaria } \\
\text { Molestias digestivas } \\
\text { Intolerancia total } \\
\text { Mareos }\end{array}$ & $\begin{array}{c}10(18.5 \%) \\
5(9.2 \% / 0) \\
1(1.8 \% / 0) \\
1(1.8 \%)\end{array}$ & 54 \\
\hline
\end{tabular}

2. Con C. + E. se estudian 29 pacientes, encontrándose $7.2 \%$ de episgastralgia, $7.2 \%$ de frigidez y $7.2 \%$ de astenia; en conjunto hubo $25.2 \%$ de trastornos; $3.6 \%$ de pacientes tuvo que dejar el tratamiento.

3. Con Diane se estudian 54 pacientes que presentaron en un $18.5 \%$ tensión mamaria, $9.2 \%$ náuseas y vómitos, y $1.8 \%$ mareos. En conjunto hubo un $31.3 \%$ de trastornos; solo en $1.8 \%$ hubo que suspender la medicación (ver Tabla No. 12).

\section{COMENTARIOS}

\section{Acción Anticonceptiva}

Hasta ahora con finalidad anticonceptiva se habían usado anovulatorios convencionales portadores de un estrógeno (etinilestradiol, mestranol, etc.) y un gestágeno (generalmente derivado de la 19 nortetosterona). No era infrecuente encontrar en la consulta de planificación familiar que algunas mujeres señalaban como efecto secundario la aparición de un discreto aumento de la pilificación, incluso en pacientes que nunca antes habían tenido esta sintomatología. 
Los preparados cuyo progestágeno era la clormadinona (con estructura similar a la del acetato de ciproterona) (28), eran, antes de aparecer esta última, los preparados idóneos para la mujer portadora de sintomatología androgénica. Pero al carecer en el mercado de este producto entra uno nuevo, el cual, aprovechando sus potentes efectos progestacional y antiandrogénico, se introduce como anticonceptivo ideal en la mujer con síntomas androgénicos (23).

En un principio existía el temor de un posible efecto antiandrogénico por su administración accidental a una mujer gestante portadora de un feto masculino, $y$ el consiguiente riesgo de feminización fetal intrauterina $(2,15)$; posteriormente se ha demostrado que dosis diarias de $2 \mathrm{mg}$ de acetato de Ciproterona (A.C.) y $0.05 \mathrm{mg}$ de Etinil estradiol (E.E.), en terapia combinada durante 21 días, tienen tan grandes márgenes de seguridad anticonceptiva como los anovulatorios convencionales y evidentes efectos beneficiosos sobre la sintomatología androgénica.

Lógicamente, la seguridad anticonceptiva y los efectos antiandrogénicos aumentan cuando aumenta la dosis de acetato de Ciproterona manteniendo la dosis de etinilestradiol, método que nosotros recomendamos en aquellas pacientes portadoras de síntomas androgénicos moderados o marcados. En nuestro material no hemos tenido ningún embarazo en 96 ciclos con Diane y 55 ciclos con C. + E., resultados que están de acuerdo con los de otros muchos autores, $(3,8,15,18,19,23,25,29,30)$.

\section{Control del Ciclo}

Cuando se usa acetato de Ciproterona a dosis continuas debe esperarse siempre la aparición de amenorrea, lo cual debe advertirse a la paciente. La que previamente a la terapéutica ya presenta alte- raciones menstruales, lo suele aceptar mejor que la paciente con menstruaciones normales; pero estás últimas, al observar la mejoría rápida de sus síntomas androgénicos, acogen esta terapéutica ya sin ningún inconveniente. Al suprimir la medicación aparece la menstruación en un breve período de tiempo, que suele oscilar entre 15 y 30 días, siendo la primera regla unas veces normal y otra de caracteres irregulares para normalizarse al ciclo siguiente.

Cuando se usa C. + E. el porcentaje de amenorrea oscila entre el 0 y el $20 \%$ según la normalidad o alteración previa del ciclo. El ciclo es normal en la mitad de los casos aproximadamente.

Con Diane lo único que llama la atención a la enferma es la disminución de la cantidad de menstruación, $7.5 \%$ a $21.5 \%$, según el tipo de regla característica de cada paciente. Resultados que superan ligeramente a los anovulatorios convencionales, pero que no crean ningún tipo de problema a la enferma (14).

\section{Acción sobre el Hirsutismo}

Dos síntomas principalmente son motivo de consulta de nuestras pacientes: la alteración menstrual, que la enferma sobrelleva con discreta preocupación, y el hirsutismo, que a veces provoca en las pacientes un gran handicap psicológico.

El exceso de vello es un síntoma objetivo con una gran carga de subjetividad, que depende de la mujer y su entorno. Esto significa que el médico debe valorar un gran espectro de presentaciones, desde una discretísima pilificación hasta una distribución pilosa francamente masculina, sin que tenga una relación directa con el grado de afectación psicológica, aspecto que es de difícil valoración.

La mujer que objetivamente presenta poca o ninguna pilificación y está con- 
vencida de tener excesiva, es muy difícil de disuadir con ningún tipo de argumento ni demostración; en consecuencia tomamos en serio a todas las pacientes $y$ antes de rechazarlas procedemos a un estudio endocrinológico mínimo y a una terapia de apoyo psicológico.

También hay que tener en cuenta una serie de variaciones fisiológicas raciales, geográficas y familiares. Nosotros, inseros como estamos en una región mediterránea, consideramos como normal una pilificación que en la región nórdica sería patológica.

La opinión respecto a los efectos beneficiosos del acetato de Ciproterona en el hirsutismo es unánime, oscilando los buenos resultados entre un 50 y un $80 \%$ $(1,4,13,14,20,31,32,34,35)$. Las diferencias en los resultados dependen de la pauta terapéutica y el grado de hirsutismo de las pacientes.

Con acetato de ciproterona a dosis continuas se obtienen mejores resultados a medida que se aumenta la dosis, existiendo diferencia significativa entre los resultados obtenidos con dosis de $50 \mathrm{mg}$ por una parte, y de 100 y 150 mg por otra.

En cambio cuando se usan dosis de $50 \mathrm{mg}$ de A.C. asociadas a E.E. se obtienen análogos resultados a los logrados con dosis continuas de 100 y 150 $\mathrm{mg}$. Parece ser como si al efecto ya de por sí beneficioso del A.C. se agregara el poder antiandrogénico del E.E.

Con respecto al Diane se aprecia una notable disminución de la respuesta con respecto a la obtenida con dosis de 100 y $150 \mathrm{mg}$ de A.C. y con C. + E., logrando en cambio mejores resultados que cuando usamos $50 \mathrm{mg}$ de A.C. en dosis continuas.
Podemos concluir que la terapéutica para el hirsutismo moderado o grave será el método continuo de altas dosis como tratamiento inicial durante $6 \mathrm{me}$ ses, continuando con tratamiento combinado de dosis bajas $(3,6,14,19,20$, $25,30,32)$.

En el hirsutismo ligero el tratamiento a utilizar es el Diane; las dosis continuas o el método combinado de altas dosis solo se usarán cuando falle la metódica anterior o cuando se necesite una respuesta rápida, (34) pues la acción del Diane solo empieza a ser manifiesta a partir de los 3 a 6 meses de iniciada la terapia.

Al comparar la suma de resultados buenos y muy buenos apreciamos que las mejores respuestas se obtienen con dosis de $100 \mathrm{mg}(81.2 \%)$ y $150 \mathrm{mg}$ $(81.7 \%)$; de otra parte, son análogas las respuesta con $50 \mathrm{mg}(57 \%)$ y el método combinado de bajas dosis $(56 \%)$, ( Tabla No. 13).

Tabla No. 13

\section{PORCENTAJES ACUMULATIVOS DE RESULTADOS BUENOS $Y$ MUY BUENOS CON LOS DISTINTOS TIPOS DE MEDICACION}

\begin{tabular}{|l|l|c|l|l|}
\hline $\begin{array}{c}\text { Tipo de } \\
\text { Tratamiento }\end{array}$ & Hirsutismo & Acné & Seborrea & Alopecia \\
\hline A.C. $50 \mathrm{mg}$. & $57 \%$ & $75 \%$ & $75 \%$ & $66.6 \%$ \\
A.C. $100 \mathrm{mg}$. & $81.2 \%$ & $100 \%$ & $91.6 \%$ & $100 \%$ \\
A.C. $150 \mathrm{mg}$. & $81.8 \%$ & $100 \%$ & $88.80 \%$ & $100 \%$ \\
C.tE. & $74.9 \%$ & $88.8 \%$ & $85 \%$ & $100 \%$ \\
Diane & $56 \%$ & $87.5 \%$ & $86.4 \%$ & $80 \%$ \\
\hline
\end{tabular}

\section{Acción sobre el Acné}

Todos los autores están de acuerdo en que el síntoma del acné es el que mejor responde a la terapéutica con A.G. (1, 
$9,10,11,19,25,26,33,36,37)$. En cambio nuestros resultados señalan que no todas las modalidades de tratamiento obtienen esos excelentes resultados, $(5$, $6,20,24)$.

El $100 \%$ de muy buenos resultados se da cuando se usa el método continuo a dosis de $150 \mathrm{mg}$; en cambio, sólo se alcanza el $81 \%$ con dosis de $100 \mathrm{mg} \mathrm{y}$ al $50 \%$ con dosis de $50 \mathrm{mg}$.

Contrariamente a lo que sucedía en el hirsutismo, las respuestas buenas con C. + E. fueron solo del $66.6 \%$.

Con método combinado de bajas dosis obtenemos mejores resultados: un $75 \%$. Sin embargo, al comparar la suma de resultados buenos y muy buenos, no apreciamos ninguna diferencia entre el método combinado de altas dosis $(88.8 \%$ ) y el Diane $(81.5 \%)$, siendo los dos superiores al tratamiento continuo con dosis de $50 \mathrm{mg}$ de A.C. $(75 \%)$.

Por lo tanto, en aquellas pacientes en que el acné predomina sobre el hirsutismo, el tratamiento de entrada será el Diane, ya que si bien existe una pequeña diferencia con el método continuo de altas dosis, los resultados se igualan rápidamente al cabo de tres meses.

\section{Acción sobre la Seborrea}

Los mejores resultados se obtienen con el método continuo a dosis de 150 mg y las demás pautas logran resultados similares.

Con la suma de resultados buenos y muy buenos, se aprecian respuestas semejantes con método continuo a dosis de 100 y $150 \mathrm{mg}$ y métodos combinados de altas y bajas dosis; siendo superiores al tratamiento con método continuo a dosis de $50 \mathrm{mg}$ (Tabla No. 13) $(20,34)$.

\section{Acción sobre la Alopecia}

Se obtienen mejores resultados con método continuo en dosis de 100 y 150 mg y método combinado de altas dosis $(100 \%)$; menos bueno con métodos combinado de bajas dosis $(80 \%)$, y sólo un $66.6 \%$ con método continuo a dosis de $50 \mathrm{mg}$ de A.C. (Tabla No. 13), $(31,37)$.

\section{Efectos secundarios}

Hubo que suprimir la medicación por intolerancia en el $4.3 \%$ de pacientes tratadas con el método continuo, el $3.6 \%$ de las tratadas con el combinado de altas dosis y el $1.8 \%$ con el combinado de bajas dosis.

Llama la atención la aparición de molestias gástricas cuando se asocia el estrógeno: $7.2 \%$ en el combinado de altas dosis y $9.2 \%$ en el combinado con bajas dosis; así como la aparición de tensión mamaria con el Diane $(18.5 \%)$.

En el tratamiento con método continuo destaca la incidencia de depresión $(8.6 \%)$ en contraste con los dos métodos combinados, resultados que coinciden todos con la mayoría de la literatura revisada, $(1,2,6,30,34,35)$.

\section{Resumen}

El acetato de Ciproterona solo o asociado con estrógenos es el producto idóneo para el tratamiento de manifestaciones androgénicas. El esquema terapéutico debe adaptarse al predominio, tiempo de evolución y severidad de unos u otros síntomas.

Cuando se trata de hirsutismo moderado o grave la pauta será la administración del método continuo a dosis altas 
durante 6 meses, seguido de método combinado de altas o bajas dosis, según el tipo de respuesta inicial.

Cuando predominan el acné y la seborrea se usará el método combinado de bajas dosis, pues si bien son mejores los resultados que se obtienen con el método continuo a dosis altas, los efectos secundarios no justifican su uso $y$ con el tiempo los resultados son semejantes. Solo cuando fracase ese método se recurrirá al método continuo con dosis altas.

Cuando predomina al alopecia el tratamiento de elección es el uso del método combinado de altas dosis, manteniendo sus efectos con método combinado de bajas dosis.

\section{A NEW ANOVULATORY ANDROGENIC}

\section{Summary}

Ciproterone acetate by itself or associated with estrogens is the most adequate product for the treatment of androgenic manifestations. Therapy must be adapted to the predominance, time of evolution and severity of the different symptoms.

When in presence of moderate or serious cases of hirsutism, the norm will be to administer the method continuouly at high doses during six months, followed by the combined method of high and low doses, according to the initial reactions.

\section{Bibliografía}

1. ABRAHAMSON L. HACKL H. SOGN. et al (1979) Klinische Erfahrungen und hormonelle Aspekte bei der Behandlung von Virilisierungs Erscheinungen bei der Frau mit Cyproteronazetat. KLIN. WOCHENSCHR. 91, 4: 126-30.

2. AYDILINK S. LACHNIT-FIXSON W. (1877) Diane Eine Gestagen-Ostrogen, Kombination mit antiandrogen wirkung. Med. Monatschr. 9: 425-29.
Whenever acne and seborrhea are predominant, the combined low-doses method should be used because, although results may be better with the continued high-doses method, side-effects do not justify its use, and results become very similar with time. High doses should be used only if the low-doses method fails.

On the other hand, when alopecia is predominant, patients should be treated with the combined high-doses method, in conjunction with a combined lowdoses method.

3. BRECKWOLDT M. TROPL R. BRAENDLE W. ROLL H. RACHEL F. (1977). Anti-androgen oral contraceptives. Int. Symp. Horm. Contracep. Utretch (Netherland) September. Excerpta Médica, 1978.

4. CAROL W. KLUGE KA. HENiPER E. KLINGER K. (1979). Erf ahrungen mit dem antiandrogen Ciproteronazetat bei der Behandlung des Hirsutismus. Zentralblt. Gynakol. 101. 8: 497-501. 
5. CORDERO LOYOLA M., BORDIU OBANZA E., LOPEZ MACIA A., MORAÑES PALLARDO E., CHARRO SAL. GADO A.L. (1981). Tratamiento del Hirsutismo idiopático con Acetato de Cyproterona y etinilestradiol: correlación clínico-hormonal. Endocrinología 28: 91. 98.

6. CREMONCINI C. LIBROIA A. VIGNA. TI E. (1976). Treatment of female hirsutism with two asociations of Cyproterone acetate and ethinyl estradiol.

Simp. Int. androgen and antiandrogen, 26-28 abril, Milán (Italia).

7. DUESTERBERG B. HUEMPEL $M$. WENDT H. (1979). Plasma levels of active ingredients after single and repeated administration of a new oral contraceptive containing $2 \mathrm{mg}$ of Cyproterone acetate and $50 \mathrm{mg}$ of ethinyl estradiol (Diane) to five young women. Acta Obstet. Gynecol. Scand. 58, suppl B: 27-31.

8. FANTA D. (1976) Acne and hirsutism form the dermatological point of view. Schriftum und Praxis 7 (2): 105-108.

9. FANTA D. (1977) Oral contraceptive in dermatology. Int. Symp. Oral contracepcion. Utretch (Nederlands): Exerpta Mé dica, 1978.

10. FANTA D. SCHNEIDER WHF. SPONA J. NEUFELD T. (1977). Die Anwendung von Antiandrogen in der Behandlung der Akne. Wiener Klinische wochenschrift. 89 (18); 622-627.

11 FANTA D. (1979) Acne basi Cliniche esperimentali della terapia ormonale. Ed. Spinger-Verlang (Viena-Milan).

12. FERRIMAN D. GALLWAY J.D. (1961). Clinical assesment of body hair growth in women. J. Clin. Endocrinol. Metab. 21: 1440 .
13. FREY H. AAKVAAG A. (1981). The treatment of essential hirsutism in women with Cyproterone acetate and ethinyl estradiol. Acta Obstet. Gynecol. Scand., 60: 295-300.

14. GALO V. ZARATE A. CANALES E.S. (1980). Gin. Obst. Mex. 48: 289-296.

15. HAMMERSTEIN J. MECKIES J. LEO ROSSBERG I. MOLTZ L. ZIELSKE F. (1975). Use of Ciproterone acetate in the treatment of acne, hirsutism, and virilism. J. Steroid. Biochem. 6 (6); 827-836.

16. HIDALGO A. BENEIT J.V. DE ANDRES F. TRELES R. SANCHEZ ALONSO, JF. BOTELLA LLUISIA J. (1981). Acetato de Ciproterona aspectos farmacológicos y terapéuticos. Acta Ginecol. 38: 209-217.

17. HUMPEL M. WENDT H. SCHULZE PE. DOGS $G=$ WEISS CHR. SPECK $U=$. (1977) Bioability and pharmacokinetics of Ciproterona acetate after oral administration of $2 \mathrm{mg}$ Cyproterone acetate in combination with $50 \mathrm{~g}$ of ethinyl estradiol to 6 young women. Contracepcion 15 (5).

18. KELLER P. J. FETZ A. SCHAR A. FLOBRSHEIM J. (1978) Behandlung von akne und seborrhoe mit antiandrogen. Schweiz. Med. Woschr. 108: 1640-1642.

19. LACHNIT FIXSON U. KAUFMAN J. (1977) Zur Beinflussung von Androgenisierungs-erscheinungen. Med. Klin. 72 (4 5): 1922-1926

20. LACHNIT FIXSON V. (1979). The deve lopment and evolution of an ovulation inhibitor (Diane) containing an antiandrogen. Acta Obstet. Gynecol. Scand. 58 (suppl 8) 33-42.

21. LAURITZEN CH. (1979). Die mannlich behaarte Frau Diagnostik und Therapie 
des Hirsutismus in der praxis. Sexualmedizin. 8 (11): 455-460.

22. LORENZO E. M. (1970). Familiar study of hirsutism. J. Clin. Endocrinol. Metab. 31: $556-561$.

23. LUDWIG E. Sobre el uso de anticonceptivos orales que contienen acetato de Ciproterona en la dermatología. 30a. Reunión de la Asociación de Dermatólogos alemanes, pgs: 172-173 Springer ed.

24. LUDWIG E. TAMM J. (1975) Neue Erkenntnisse auf dem Gebiet der androgenetischen alopecia Akt. Dermatol. 1:, 219-227.

25. LUDWIG E. MEINHOF W. ZAUN H = (1977) Zur Therapie androgen-abhengiger Hauterscheinungen mit CyproteronazetatUstrogen-Kombinationem, Akt. Dermatol. 3: 201-209.

26. MAUSS J. (1981). Cyproterone therapy of papulo pustular rosacea in women Hautzart. 32 (2), 94-95.

27. MILLET A. BONILLA MUSOLES F. TORTAJADA M. (1975) Los test dinámicos. Rev.- Esp. Obstet. Ginecol. 34: 107-111.

28. MILLET A. FERRER J. (1977) Androcur: un potente antiandrógeno. Rev. Esp. Obstet. Ginecol. 34: 360-365.

29. MILLET A. FERRER J. CORTAJADA M. GIL F. (1977). Tratamiento del Hirsu'tismo con Androcur. Rev. Esp. Obstet. Ginecol. 34: 360-365.

30. MOLTZ L. MEICKES J. HAMMERSTEIN J. (1979) Die Kontraceptive Betreung, Androgenisierter frauen mit einem nie- drig Dosierten Cyproteronacetathaltigen Einphasenpreparat. Dtsch. Woschr. 104: 1376-1382.

31. MOLTZ L. SCHWARTZ U. HAMMERSTEIN J. (1981). Hyperandrogenische Ovarialinsuffizienz. Gynaekologe 14 (2): 119 130.

32. NEUMANN F. (1980). AntiandrógeneWann sind sie Heute indiziert, Moderne, Medizin 2: 17-24.

33. RIITA p. PEKKA Y. ANTII T. (1978). Treatment of acne with Ciproterone acetate and ethniyl estradiol Acta Dermatovener (Stockholm) 58: 449-454.

34. SCHMIDT-ELMENDORFF H. STEYER M. (1977). Klinische Erfahrungen mit einem niedrig dosierten Antiandrogen (cyproteronazetat) und ethiniloestradiol bei der Frauen mit Virilisierungsercheinungen. Geburtsh. Frauenheilk 37 (4) 295-303.

35. YLOSTAO P. LAAKSO L. VIINIKKAL. YLIKOR KALA O. VIHKO R. (1981). Cyproterone acetate in the treatment of hirsutism. Acta Obstet. Gynecol. Scand. 60: 399-401.

36. ZAUN H. und LUDWIG E. (1978). Dermatologische indikatsionsstellung zur antiandrogen Behandlung. Z. Hautkr. 53 (21): 759-765.

37. ZAUND H. (1979). Fortschritte der praktischen Dermatologie und Venerologie Vol. 9 p. 374. Ed. von O. BraunFelco y H.H. Wolff. Springer-Verlag Berlin, Heidelberg. 